\title{
Opioid Overdose Ambulance Runs: How Wisconsin Uses Free Text Data
}

\author{
Ashley Bergeron ${ }^{1}$, Jennifer Broad ${ }^{\star 1}$, Dr. Ousmane Diallo1, Gayatri Raol1,2 \\ and Milda Aksamitauskas ${ }^{1}$
}

${ }^{1}$ Office of Health Informatics, Wisconsin Department of Health Services, Madison, WI, USA; ${ }^{2}$ Council of State and Territorial Epidemiologists, Madison, WI, USA

\section{Objective}

1. Develop an understanding of the benefits and challenges of analyzing free text fields on a population level.

2. Observe how a complex surveillance definition can be created from free text fields.

3. Observe how an ambulance data system can be used to describe the opioid epidemic.

\section{Introduction}

In 2016, twelve states received Center for Disease Control and Prevention (CDC) Enhanced State Opioid Overdose Surveillance grants. The purpose of the grant is to explore enhanced data sources to track nonfatal opioid overdoses. One data source is ambulance runs. Wisconsin collects ambulance run information within the Wisconsin Ambulance Runs Data System (WARDS). Around 84\% of all Wisconsin administrative services report into this electronic system. This is a timely, robust data system that has not been used previously to examine drug overdoses and presents an analytical challenge as it contains many free text fields.

\section{Methods}

Wisconsin's ambulance data system is robust, well-populated, and includes the majority of Emergency Medical Services (EMS) within the state. The analytic challenge with this data is that most of the reported fields are free text, which can be difficult to analyze on a population level. Wisconsin created a case definition using SAS regular expressions to take advantage of the free text fields. A combination of fields (chief complaint, secondary complaint, medications given, and the EMS narrative) were used to determine if the ambulance run was due to an opioid overdose. It was necessary to create a definition that used a diverse combination of phrases as free text fields are prone to spelling errors and there are many phrases used to identify opioid overdoses. It was also necessary to create a definition for unwanted phrases that signal a false positive, for example, "withdrawal".

\section{Results}

Wisconsin's opioid definition uses regular expressions to search for the words "heroin", "opioid", "narcan", or "methadone" (including various spellings). The overdose definition searches for words and phrases like "drug abuse", "drug use", "poisoning", "drug ingestion", and "overdose". The medication administered fields are examined for "narcan". In Wisconsin, the medication is listed every time it is used, so it is possible to determine the number of times Narcan was administered to a single person as well as how many ambulance runs used at least one dose of Narcan. False positives are identified with words and phrases like "withdrawal", "detox", and if Narcan was given but there is no indication that the ambulance run was due to drugs. From January 2016 - June 2017, Wisconsin had over 917,000 ambulance runs for people aged 11 years and older. We excluded nonemergency ambulance runs, like medical transports, and so our final denominator was 627,536 runs $(32 \%$ of all runs were classified as non-emergencies). Suspected opioid overdoses were determined to be
$1 \%$ of emergency ambulance runs. Narcan was administered in a total of 5,900 runs and the false positive flag picked up 10,399 runs that may not have been due to suspected opioid overdoses. Applying all of these components together, it was determined that in Wisconsin from January 2016 - June 2017, there were 4,041 emergency ambulance runs due to suspected, unintentional opioid overdoses for people 11 years and older (rate of 6 per 1,000 people).

\section{Conclusions}

The use of regular expressions enables Wisconsin to extend analyses to data systems that contain robust information within free text fields. Within Wisconsin, this has been utilized to enhance opioid overdose surveillance with the use of a rapid data system previously not examined. Ambulance run information is a valuable resource to Wisconsin with the opioid epidemic. By creating case definitions with free text fields, we can quantify ambulance runs on a population level and create linkable analytic data sets to provide a more complete picture of the health of Wisconsin.

\section{Keywords \\ Opioid; Surveillance; Ambulance; Free Text}

\section{Acknowledgments}

This abstract was supported by the Grant or Cooperative Agreement Number, U17 CE924885, funded by the Centers for Disease Control and Prevention. Its contents are solely the responsibility of the authors and do not necessarily represent the official views of the Centers for Disease Control and Prevention or the Department of Health and Human Services.

*Jennifer Broad

E-mail: jennifer.broad@dhs.wisconsin.gov 